\title{
Ajuste de dose dos medicamentos de acordo com a função renal em um hospital universitário
}

\author{
Dose adjustment of medicines according to renal function in \\ a university hospital
}

Recebido em: 01/02/2021 Aceito em: $13 / 07 / 2021$
Gisele Araújo RODRIGUES ${ }^{1}$; Jessica Wedna da Silva ALVES ${ }^{2}$; Regina Meira Lima de SOUZA ${ }^{1}$; Laís Silva de VASCONCELOS ${ }^{1}$; Celuane Alves MOURA ${ }^{1}$; Ricardo BRANDÃ ${ }^{2}$; Francisca Sueli Monte MOREIRA ${ }^{2}$ ${ }^{1}$ Hospital das Clínicas; ${ }^{2}$ Departamento de Farmácia. Universidade Federal de Pernambuco. Av. Prof. Moraes Rego, 1235, Cidade Universitária, CEP 50670-901. Recife, PE, Brasil. E-mail: giaraujo208@gmail.com

\section{ABSTRACT}

This study evaluated the need for dose adjustment of medications according to the renal function of patients in the medical clinic in a university hospital in the State of Pernambuco, Brazil. The study follows a cross-sectional design. The prescriptions of patients hospitalized from September 2019 to February 2020 were analyzed. The study follows a retrospective longitudinal design. The prescriptions of patients hospitalized from September 2019 to February 2020 were analyzed. During this period, 245 patients had their prescriptions evaluated. Of these, 45 patients (18.3\%) were excluded due to the absence of creatinine information and $152(62.0 \%)$ because they did not require adjustment of the dose of medication because renal function was within normal parameters. The remaining 48 patients were included in the final study, because they presented altered renal function and used renal excreted drugs. It was found that $60.4 \%$ received at least one prescription during hospitalization with an unadjusted dose of medication according to renal function. Among the drugs with unadjusted doses, antimicrobials were the most frequent. This study warns of the importance of monitoring renal function, especially when prescription drugs excreted renally.

Keywords: renal elimination; creatinine; drug prescriptions; medication error; glomerular filtration rate.

\section{RESUMO}

O presente estudo avaliou a necessidade de ajuste de dose dos medicamentos de acordo com a função renal dos pacientes da clínica médica em um hospital universitário do Estado de Pernambuco. O estudo seguiu delineamento transversal. Foram analisadas as prescrições dos pacientes internados no período de setembro de 2019 a fevereiro de 2020. Nesse período, 245 pacientes tiveram suas prescrições avaliadas. Destes, 45 pacientes $(18,3 \%)$ foram excluídos devido à ausência de informações sobre creatinina e $152(62,0 \%)$ por 
não necessitarem ajuste da dose de medicamento, porque a função renal estava dentro dos parâmetros de normalidade. Os 48 pacientes restantes foram incluídos no estudo final, pois apresentavam função renal alterada e utilizavam medicamentos excretados por via renal. Dos pacientes, 60,4\% receberam, durante a internação, pelo menos uma prescrição com dose de medicamento não ajustado de acordo com a função renal. Dentre os medicamentos com doses não ajustadas, os antimicrobianos foram os mais frequentes. Este estudo alerta para a importância do acompanhamento da função renal, principalmente quando prescritos medicamentos excretados via renal.

Palavras-chave: eliminação renal; creatinina; prescrições de medicamentos; erro de medicação; taxa de filtração glomerular.

\section{INTRODUÇÃO}

Pacientes hospitalizados utilizam, geralmente, um grande número de medicamentos, em decorrência de suas complexas condições clínicas. Quando se trata de medicamentos excretados por via renal, sendo utilizados por pacientes que apresentam disfunção renal importante, podem ser necessários ajustes de dose para evitar-se toxicidade e eventos adversos relacionados a medicamentos (EAM) (1). O adequado ajuste de doses dos medicamentos depende da correta avaliação da função renal dos pacientes, especialmente quando são fármacos excretados na forma inalterada na urina, que possuem faixa terapêutica estreita, com metabólitos ativos excretados pelo rim ou que são nefrotóxicos (2). A falta de ajuste das doses dos medicamentos para indivíduos que apresentam comprometimento da função renal pode acarretar redução da depuração dos fármacos, expondo o paciente ao acúmulo dos mesmos no organismo e, consequentemente, ao maior risco de toxicidade e EAM. Além disso, nesses pacientes, as ligações às proteínas plasmáticas podem ser consideravelmente reduzidas, podendo assim repercutir em processos farmacocinéticos de distribuição e de excreção dos fármacos (3).

Dessa forma, a avaliação da função renal dos pacientes por meio da estimativa da taxa de filtração glomerular (TFG) a partir da mensuração da creatinina sérica, é considerada de grande importância para a segurança da terapia medicamentosa. A creatinina sérica é o marcador de diagnóstico mais usualmente utilizado para análise da função renal na prática clínica, sendo utilizada para deter- minar a necessidade de ajuste de dose por meio de equações matemáticas (1). Tais equações são baseadas na dosagem da creatinina sérica, na idade, gênero, superfície corpórea e etnia. As fórmulas mais frequentemente utilizadas são Cockcroft-Gault (CG), Modification of Diet in Renal Disease (MDRD), Chronic Kidney Disease Epidemiology Collaboration (CKD-EPI) (4).

$\mathrm{Na}$ prática clínica hospitalar o ajuste de dose dos medicamentos prescritos de acordo com a função renal ainda é bastante negligenciado. Em um estudo realizado com pacientes hospitalizados, com comprometimento da função renal, 15\% a 75\% dos medicamentos prescritos necessitavam ter suas doses ajustadas, porém os ajustes não foram realizados e doses inadequadas foram administradas (5).

Dentre as estratégias para redução de danos ao paciente, pode ser destacada a atuação do farmacêutico clínico, que por meio de revisões da prescrição e do acompanhamento da farmacoterapia, pode colaborar com a equipe multiprofissional e reduzir os EAM. Um estudo de Lazaryan e cols (2019) demonstrou que as intervenções farmacêuticas relacionadas à correção de dose dos medicamentos têm contribuído para melhorar a segurança do paciente e reduzir o tempo de hospitalização (6). A participação do farmacêutico na correção de dose dos medicamentos excretados por via urinária tem contribuído de forma positiva na função renal do paciente (7).

Em função disto, o presente estudo tem como objetivo avaliar a necessidade de ajuste de dose dos medicamentos de acordo com a função renal dos pacientes da clínica médica em um hospital universitário do estado de Pernambuco. 


\section{MÉTODO}

Trata-se de um estudo transversal retrospectivo, realizado no período de março a agosto de 2020 no Hospital de Ensino da Universidade Federal de Pernambuco (UFPE) O hospital tem 418 leitos e a Enfermaria da Clínica Médica, possui 30 leitos para internação, com média de 40 internamentos por mês.

Foram incluídas no estudo as prescrições dos pacientes maiores de 18 anos de idade, de ambos os sexos, hospitalizados no período de setembro de 2019 a fevereiro de 2020 na Enfermaria de Clínica Médica e que utilizavam medicamentos que necessitam do ajuste de dose para a função renal. Foram excluídos os pacientes que não realizaram avaliação laboratorial da creatinina sérica ou que foram avaliados quanto à função renal, mas utilizaram apenas medicamentos que não necessitavam de ajuste renal de dose.

A primeira etapa do estudo consistiu no levantamento dos medicamentos padronizados que precisassem de ajuste de dose ou da frequência da administração, os quais passaram a ser classificados como "medicamentos com risco renal". Esta classificação foi realizada a partir de revisão das bases de dados: Micromedex ${ }^{\circledR}$, UpToDate ${ }^{\circledR}$ e da Tabela de correção de doses de antibióticos para função renal do HC/UFPE.

A segunda etapa do estudo consistiu na avaliação retrospectiva de todas as prescrições dos pacientes hospitalizados na Clínica Médica que atendiam aos critérios de inclusão, sendo observada a presença de algum medicamento com risco renal e a realização do exame de creatinina sérica. Baseado nestas informações, foi calculado, para cada paciente, o Clearence de creatinina, por meio da calculadora CKD-EPI, preconizada pela equipe médica para avaliar a função renal do paciente. A prescrição foi então classificada como Prescrição Potencialmente Inapropriada (PPI), quando continha pelo menos um medicamento com risco renal cujo ajuste de dose não havia sido realizado conforme as bases de dados citadas anteriormente. Para os antimicrobianos, somente foi considerado como ajuste incorreto quando a não realização do ajuste ocorreu após a dose de ataque ( $>24 \mathrm{~h})$, conforme a recomendação do protocolo assistencial do Hospital (manutenção da dose de ataque).
Foram avaliadas ainda as variáveis sociodemográficas (sexo, raça e idade), as variáveis relacionadas ao período de internamento do paciente, aos exames de creatinina realizados no período de hospitalização, os medicamentos de risco renal e os medicamentos prescritos com ajuste de dose adequado. As variáveis foram coletadas por meio da prescrição eletrônica e do programa MasterTools ${ }^{\circledR}$.

As variáveis coletadas foram tabuladas em planilha do Microsoft Office Excel ${ }^{\circledR}$ 12.0. Para análise estatística foi utilizado o software SPSS 19.0. Para estatística descritiva foram analisadas medidas de tendência central (média) e dispersão (desvio padrão).

O estudo foi aprovado pelo Comitê de Ética em Pesquisa (CEP) do HC/UFPE, sob o parecer $\mathrm{n}^{\circ} 3.919 .556$.

\section{RESULTADOS E DISCUSSÃO}

Durante o estudo, no período de 6 meses, 245 pacientes da Enfermaria da Clínica Médica tiveram suas prescrições avaliadas quanto ao uso de medicamentos com risco renal e a mensuração da creatinina sérica. Destes, foram excluídos 45 indivíduos por não terem realizado exame de creatinina e 152 por apresentarem a função renal dentro dos parâmetros da normalidade. Os 48 pacientes restantes foram incluídos no estudo.

As características sociodemográficas e clínicas dos pacientes avaliados são descritas na (Tabela 1). Para aqueles que precisavam de ajuste de dose, notou-se um predomínio do sexo masculino, com média de idade de 60,9 anos. Na amostra predominou a raça parda. $\mathrm{O}$ tempo médio de internamento foi de 16 dias. A média de dias de exame de creatinina realizado durante a hospitalização foi 10,5 17,08 dias.

Entre aqueles que receberam medicamentos que exigem ajuste de dose, $39,6 \%(n=19)$ tiveram todos os seus medicamentos ajustados corretamente; enquanto $60,4 \%(n=29)$ receberam, durante a hospitalização, pelo menos uma prescrição com medicamento não ajustado para a função renal. Dos pacientes que não tiveram a correção, $41,7 \%$ $(\mathrm{n}=20)$ fizeram uso de pelo menos um medicamento com risco renal, sem adequada realização 
Tabela 1. Características sociodemográficas e clínicas dos pacientes internados na Enfermaria de Clínica Médica que precisavam de ajuste de dose no período de setembro de 2019 a fevereiro de 2020.

\begin{tabular}{|l|l|}
\hline Características & Pacientes que precisavam de ajuste de dose (n=48) \\
\hline Sexo masculino (n, \%) & $25(52,1 \%)$ \\
\hline Idade, anos (média, DP) & $60,9 \pm 20,0$ \\
\hline Raça pardo (n, \%) & $42(87,5 \%)$ \\
\hline Dias internado (média, DP) & $16,2 \pm 9,23$ \\
\hline Dias fez creatinina (média, DP) & $10,5 \pm 7,08$ \\
\hline Dias sem creatinina (média, DP) & $5,12 \pm 3,71$ \\
\hline Número de Medicamentos que precisavam de ajuste & $1,47 \pm 0,77$ \\
\hline \multicolumn{1}{|c|}{ Frequência de medicamentos que precisavam de ajuste $(\mathbf{n}, \%)$} \\
\hline Um medicamento & $20(41,7 \%)$ \\
\hline Dois medicamentos & $8(16,7 \%)$ \\
\hline Três medicamentos & $1(2,1 \%)$ \\
\hline
\end{tabular}

da correção de dose; $16,7 \%$ fez uso de dois; e $2,1 \%$ paciente fez uso de três medicamentos nefrotóxicos com dose considerada inadequada.
Os medicamentos mais prescritos que precisavam ter suas doses ajustadas são apresentados na Tabela 2. Dentre estes, a classe dos antimicrobianos

Tabela 2. Frequência de medicamentos que necessitavam ter doses ajustadas de acordo com a função renal dos pacientes na Enfermaria de Clínica Médica no período de setembro de 2019 a fevereiro de 2020.

\begin{tabular}{|c|c|c|c|c|}
\hline Medicamentos & $\begin{array}{l}\text { Classificação } \\
\text { ATC }\end{array}$ & $\begin{array}{l}\text { Precisava de ajuste } \\
\text { de dose }(n=67)\end{array}$ & $\begin{array}{l}\text { sem correção } \\
\text { de dose }(n=38)\end{array}$ & $\begin{array}{l}\text { com correção } \\
\text { de dose }(n=29)\end{array}$ \\
\hline Meropenem (n, \%) & $\mathrm{J01DH}$ & $16(23,9 \%)$ & $9(23,7 \%)$ & $7(24,1 \%)$ \\
\hline Piperacilina+tazobactam (n, \%) & J01CR & $12(17,9 \%)$ & $7(18,4 \%)$ & $5(17,2 \%)$ \\
\hline Ciprofloxacino (n, \%) & J01MA & $6(9,0 \%)$ & $3(7,9 \%)$ & $3(10,3 \%)$ \\
\hline Morfina (n, \%) & N02AA & $5(7,5 \%)$ & $3(7,9 \%)$ & $2(6,8 \%)$ \\
\hline Hidralazina (n, \%) & C02DB & $4(6,0 \%)$ & $2(5,3 \%)$ & $2(6,8 \%)$ \\
\hline Ampicilina+tazobactam (n, \%) & J01CR & $3(4,5 \%)$ & $3(7,9 \%)$ & - \\
\hline Enalapril (n, \%) & CO9AA & $3(4,5 \%)$ & - & $3(10,3 \%)$ \\
\hline Atenolol (n, \%) & CO7AB & $2(3,0 \%)$ & - & $2(3,0 \%)$ \\
\hline Sulfametoxazol+trimetropim (n, \%) & J01EE & $2(3,0 \%)$ & - & $2(3,0 \%)$ \\
\hline Teicoplanina (n, \%) & J01XA & $2(3,0 \%)$ & $2(5,3 \%)$ & - \\
\hline Metronidazol (n, \%) & J01XD & $2(3,0 \%)$ & $1(2,6 \%)$ & $1(3,4 \%)$ \\
\hline Tramadol (n, \%) & N02AX & $2(3,0 \%)$ & $2(5,3 \%)$ & - \\
\hline Anfotericina B desoxicolato ( $n, \%)$ & J02AA & $1(1,5 \%)$ & $1(2,6 \%)$ & - \\
\hline Fluconazol (n, \%) & J02AC & $1(1,5 \%)$ & $1(2,6 \%)$ & - \\
\hline Gentamicina $(n, \%)$ & J01GB & $1(1,5 \%)$ & - & $1(1,5 \%)$ \\
\hline Micofenolato de mofetila $(n, \%)$ & L04AA & $1(1,5 \%)$ & $1(2,6 \%)$ & - \\
\hline Pentoxifilina (n, \%) & CO4AD & $1(1,5 \%)$ & $1(2,6 \%)$ & - \\
\hline Captopril (n, \%) & CO9AA & $1(1,5 \%)$ & - & $1(1,5 \%)$ \\
\hline Metoclopramida (n, \%) & A03FA & $1(1,5 \%)$ & $1(2,6 \%)$ & - \\
\hline Vancomicina $(n, \%)$ & J01XA & $1(1,5 \%)$ & $1(2,6 \%)$ & - \\
\hline
\end{tabular}


foi a mais frequentemente prescrita sem a realização da correção de dose.

O presente estudo identificou que 60,4\% $(n=29)$ dos pacientes que utilizavam medicamentos com risco renal e que apresentavam função renal fora dos parâmetros da normalidade receberam, durante a internação, pelo menos uma prescrição com algum medicamento cuja dose não foi devidamente ajustada.

Em um estudo realizado por Decloedt e cols (2010) no contexto hospitalar com 69 pacientes que precisavam de ajuste para função renal, apenas $12 \%$ tiveram o ajuste realizado adequadamente; $29 \%$ tiveram alguns medicamentos ajustados e $59 \%$ não tiveram o ajuste realizado (8). O estudo de O'shaughnessy e cols (2017) identificou prevalência de $65,5 \%$ de prescrição potencialmente inapropriada (9).

O presente estudo identificou frequência diferente das demais relatadas anteriormente, provavelmente por utilizarmos fontes de informação diferentes das adotadas e pela inclusão de todas as prescrições dos pacientes com a mensuração da creatinina.

A prescrição de medicamentos em doses elevadas ou em subdoses, em decorrência de não ajuste baseado na função renal do paciente (10), são considerados problemas relacionados a medicamentos (10). Doses abaixo da faixa terapêutica podem acarretar redução da eficácia terapêutica (11), enquanto dose prescrita acima da dose recomendada em pacientes com diminuição da depuração renal, acarreta aumento das concentrações séricas implicando em maior risco de toxicidade (12). É, portanto, de suma importância considerar a função renal do paciente quando são prescritos medicamentos excretados por via renal (13).

Neste estudo foi observado que grande parte dos pacientes hospitalizados no período estudado tinha mais de 60 anos. Tal achado reforça a necessidade de maior acompanhamento da função renal, principalmente quando há prescrição de medicamento excretado pela via renal (5). De acordo com Okoro e cols (2019), os idosos são também mais predispostos a utilizarem medicamentos nefrotóxicos (14) e segundo Altunbas e cols (2016), devido à redução na TFG, há uma maior probabilidade de prescrição inadequada (15). É importante destacar ainda, que alterações na TFG no idoso podem ser tardiamente identificadas, devido a mudanças fisiológicas relacionadas ao envelhecimento como a diminuição da massa muscular, mantendo assim a creatinina sérica dentro dos parâmetros normais, mesmo quando o paciente já apresenta função renal reduzida (5).

A média de tempo de internação no presente estudo entre aqueles com alteração da função renal foi 16,2 $\pm 9,23$ dias, achados condizentes com o nosso resultado foram descritos por Rodrigues e Bertoldi (2010) na pesquisa de perfil de utilização de antimicrobianos em hospital privado com média do tempo de internação de $14 \pm 17,7$ dias (16). Nessa investigação, a classe de medicamentos mais prevalente nas PPI foi a dos antimicrobianos. Dentre estes, os mais frequentes foram, meropenem, piperacilina+tazobactam e ciprofloxacino, respectivamente.

Em pesquisa do tipo observacional com pacientes hospitalizados que apresentavam doença renal crônica os antibióticos também foram a classe de fármacos mais relacionados a PPI (9). Da mesma forma, Dewitt e cols (2016), num estudo coorte em uma Unidade de Terapia Intensiva (UTI), identificou maior prevalência de prescrição de dose incorreta com os antibióticos piperacilina+tazobactam e ciprofloxacino (17). Segundo Molmar e cols (2020) os antibióticos foram a classe de medicamentos mais associada ao risco de PPI para pacientes renais agudos ou crônicos (18). É importante ressaltar, que antimicrobianos, anti-hipertensivos, quimioterápicos, imunossupressores e anti-inflamatórios são classes de medicamentos frequentemente associados ao surgimento da nefrotoxicidade (19).

Neste estudo, o meropenem foi o antimicrobiano mais frequentemente prescrito e para o qual não foi realizado o ajuste de dose corretamente $(13,4 \%)$. A piperacilina+tazobactam, também da classe dos beta-lactâmicos, foi o segundo medicamento mais prescrito, porém foi o mais ajustado, quando comparado ao meropenem. Um estudo conduzido no Japão comparando a piperacilina+tazobactam ao cefepime, mostrou que a monoterapia da piperacilina+tazobactam está mais associada a maior incidência da LRA (20).

Dos pacientes admitidos no período do estudo, 45 não foram incluídos na amostra, devido à 
não avaliação da função renal. Desses, 53,3\% eram pacientes idosos e por ausência de solicitação de creatinina sérica, não foi possível realizar o cálculo da TFG e julgar quanto a necessidade de correção de dose dos medicamentos. A média de permanência hospitalar desses pacientes foi de 4,89 (dias), com no mínimo 1 dia e no máximo 30 dias de internamento. Portanto, consideramos que a duração da internação permitiria a avaliação da função renal por meio da solicitação do exame laboratorial de creatinina.

Os resultados deste estudo necessitam ser interpretados com cautela, uma vez que a natureza retrospectiva da coleta de dados pode ter impactado na incompletude de informações, e especialmente devido à ausência do resultado laboratorial da creatinina que impactou em exclusão de boa parte da população elegível ao estudo. Apesar dessas limitações, o estudo possibilitou estabelecer o conhecimento acerca dos medicamentos mais frequentemente prescritos que necessitam de correção de dose para pacientes da Enfermaria da Clínica Médica e identificou múltiplas oportunidades para o fortalecimento de ações que poderão otimizar a segurança do uso de medicamentos por pacientes com alterações da função renal.

\section{CONCLUSÃO}

Foi frequente a ocorrência de medicamentos com risco renal sem ajuste de dose adequado para pacientes com alguma alteração da função renal. A principal classe de medicamentos que necessitava de ajuste de dose foi a dos antimicrobianos; dentre esses, os mais frequentemente prescritos foram meropenem, piperacilina+tazobactam e ciprofloxacino. Foi observado que uma parcela importante dos pacientes não teve, durante o internamento, a solicitação de exames que avaliassem a função renal.

Espera-se que o presente estudo possa contribuir para a consolidação de medidas que venham a ser implementadas visando a segurança do paciente, como, a inclusão do farmacêutico clínico na equipe, para efetivar o acompanhamento farmacoterapêutico dos pacientes que possuem diminuição da depuração renal, visto que o ajuste de dose é de suma valia para garantir a eficácia da farmacoterapia e redução de risco a saúde do paciente.

\section{REFERÊNCIAS}

1. Karpa K, Difelice R. Drug toxicity in kidney disease: A standardized patient case for clerkship students. Mededportal. 2016;12:1-7. DOI: 10.15766/mep 23748265.10464

2. Khanal A, Peterson GM, Castelino RL, Jose MD. Renal drug dosing recommendations: evaluation of product information for brands of the same drug. Int Med J. 2014;4(6):591-596. DOI: 10.1111/imj.12446

3. Getachew H, Tadesse Y, Shibeshi W. Drug dosage adjustment in hospitalized patients with renal impairment at Tikur Anbessa specialized hospital, Addis Ababa, Ethiopia. BMC Nephrol. 2015;16(1):1-9. DOI: 10.1186/ s12882-015-0155-9

4. Brito TNS, Oliveira ARA, Silva AKC. Glomerular filtration rate estimated in adults: characteristics and limitations of equations used. Rev Bras Anal Clin. 2016;1(48):7-12

5. Dörks M, Allers K, Schmiemann G, Herget-Rosenthal $\mathrm{S}$, Hoffmann F. Inappropriate medication in nonhospitalized patients with renal insufficiency: a systematic review. J Am Geriatr Soc. 2017;65(4): 853-862. DOI: $10.1111 /$ jgs. 14809
6. Lazaryan M, Abu-Kishk I, Rosenfeld-Yehoshua N, Berkovitch S, Toledano M, Reshef I, Kanari T, ZivBaran T, Berkovitch M. Pharmacist remote review of medication prescriptions for appropriateness in pediatric intensive care unit. Front Pharmacol. 2019; 7(243):1-7. DOI: 10.3389/fphar.2016.00243

7. Cabello-Muriel AJJ, Urbieta-Sanz E, Iniesta-Navalón C. Effectiveness of pharmacist intervention in patients with chronic kidney disease. Int J Clin Pharm. 2014;36(5):896903. DOI: 10.1007/s11096-014-0001-3

8. Decloedt E, Leisegang R, Blockman M, Cohen K. Dosage adjustment in medical patients with renal impairment at Groote Schuur Hospital. S Afr Med J. 2010;100(5):304-306. DOI 10.7196/samj.3955

9. O'shaughnessy M, Allen N, O'Regan J, Payne-Danson E, Mentre L, Davin D, Lavin P, Grimes T. Agreement between renal prescribing references and determination of prescribing appropriateness in hospitalized patients with chronic kidney disease. QJM. 2017;110(10):623628. DOI: $10.1093 /$ qjmed/hcx086 
10. Pfister B, Jonsson J, Gustafsson M. Drug-related problems and medication reviews among old people with dementia. BMC Pharmacol Toxicol. 2017; 18(52):3-11

11. Saad R, Hallit S, Chahine B. Evaluation of renal drug dosing adjustment in chronic kidney disease patients at two university hospitals in Lebanon. Pharm Pract. 2019;17(1):1-6. DOI: 10.18549/PharmPract.2019.1.1304

12. Maxson R. Medications in kidney disease. The journal for nurse practitioners. 2017; 13(10): 687-692. DOI: 10.1016/j.nurpra.2017.07.023

13. Castelino RL, Saunder T, Kitsos A, Peterson GM, Jose M, Wimmer B, Khanam M, Bezabhe W, Stankovich J, Radford J. Quality use of medicines in patients with chronic kidney disease. BMC Nephrol. 2020;21(1):1-9. DOI: $10.1186 / \mathrm{s} 12882-020-01862-1$

14. Okoro RN, Farate VT. The use of nephrotoxic drugs in patients with chronic kidney disease. Int J Clin Pharm. 2019;41(3):767-775. DOI: 10.1007/s11096-019-00811-9

15. Altunbas G. Yazc M, Solak Y, Gul EE, Kayrak M, Kaya Z, Akilli H, Aribas A, Gaipov A, Yazc R, Ozdemir K. Renal drug dosage Adjustment According to Estimated Creatinine Clearance in Hospitalized Patients With Heart Failure. Am J Ther. 2016;23(4):1-5. DOI: 10.1097/01. mjt.0000434042.62372.49
16. Rodrigues FA, Bertoldi AD. Perfil da utilização de antimicrobianos em um hospital privado. Ciênc Saúde Colet. 2010;15(1):1239-1247. DOI: $10.1590 / \mathrm{S} 1413-$ 81232010000700033

17. Dewitt KM, Weiss SJ, Rankin S, Ernst A, Sarangarm P. Impact of an emergency medicine pharmacist on antibiotic dosing adjustment. Am J Emerg Med. 2016;34(6):980-984. DOI: 10.1016/j.ajem.2016.02.004

18. Molnar AO, Bota S, Jeyakumar N, McArthur E, Battistella M, Garg AX, Sood MM, Brimble KS. Potentially inappropriate prescribing in older adults with advanced chronic kidney disease. PlosOne. 2020;8(15):1-16. DOI: 10.1371/journal.pone. 0237868

19. Downes KJ, Hayes M, Fitzgerald JC, Pais GM, Liu J, Zane NR, Goldstein S, Scheetz MH, Zuppa AF. Mechanisms of antimicrobial-induced nephrotoxicity in children. J Antimicrob Chemother. 2020;1(75):1-13. DOI: $10.1093 / \mathrm{jac} / \mathrm{dkz} 325$

20. Kadomura S, Takekuma Y, Sato Y, Sumi M, Kawamoto K, Itoh T, Sugawara M. Higher incidence of acute kidney injury in patients treated with piperacillin/tazobactam than in patients treated with cefepime: a single-center retrospective cohort study. J Pharm Health Care Sci. 2019;5(13):1-8. DOI: 10.1186/s40780-019-0142-6 